\title{
Development of mixed convection flow over a vertical plate due to an impulsive motion
}

\author{
M. Kumari, G. Nath
}

Abstract The development of the mixed convection flow of an incompressible laminar viscous fluid over a semiinfinite vertical plate has been investigated when the fluid in the external stream is set into motion impulsively, and at the same the surface temperature is suddenly raised from its ambient temperature. The problem is formulated in such a way that at time $t=0$, it reduces to Rayleigh type of equation and as time $t \rightarrow \infty$, it tends to Blasius type of equation. The scale of time has been selected such that the traditional infinite region of integration becomes finite which significantly reduces the computational time. The nonlinear coupled singular parabolic partial differential equations governing the unsteady mixed convection flow have been solved numerically by using an implicit finitedifference scheme. The surface shear stress and the heat transfer increase or decrease with time when the buoyancy parameter is greater or less than a certain valve. There is a smooth transition from the initial steady state to the final steady state. The skin friction and heat transfer for the constant heat flux case are more than those of the constant wall temperature case. Also they increase with the buoyancy force.

\section{1}

\section{Introduction}

The mixed convection flow occurs in several industrial and technical applications which include nuclear reactors cooled during emergency shutdown, electronics devices cooled by fans, heat exchangers placed in a low velocity environment, and solar central receivers exposed to wind currents. In the study of fluid over heated or cooled surfaces, it is customary to neglect the effect of the buoyancy forces when the flow is horizontal. However for vertical or inclined surfaces, the buoyancy force modifies the flow field and hence the heat transfer rate. Therefore, it is not possible to neglect the effect of buoyancy forces for vertical or inclined heated or cooled surfaces. In recent years,

M. Kumari ( $\square)$, G. Nath Department of Mathematics, Indian Institute of Science, Bangalore-560 012, India

E-mail: mkumari@math.iisc.ernet.in much attention has been paid to develop efficient energy systems.

Sparrow et al. [1] were the first to study the effect of the buoyancy forces on the forced convection flow over a vertical flat plate and obtained the similarity solution when both the non-isothermal wall temperature and non-uniform external flow vary with the streamwise distance from the leading edge of the plate in a particular way. Subsequently, Sparrow and Gregg [2] obtained nonsimilar solution of the combined forced and free convection flow over a vertical surface by using the series expansion method. Szewczyk [3] considered the effects of the buoyancy forces on a forced convection flow as well as the forced convection effects on a purely free convection flow on a vertical flat plate. Solutions of the boundary layer equations were obtained by expanding the stream function and temperature function in terms of the buoyancy parameter. Merkin [4] investigated the mixed convection on the boundary layer flow over a semi-infinite vertical flat plate when the buoyancy forces aid and oppose the boundary layer development. In the former case, two series solutions were obtained, one of which was valid near the leading edge and the other was valid asymptotically. In the region where the series solutions are not valid, the equations were solved numerically. Oosthuizen and Hart [5] carried out a numerical study of the mixed convection flow over a heated vertical surface. The mixed convection flow over an inclined surface for both constant wall temperature and heat flux cases was studied by Mucoglu and Chen [6]. The boundary layer equations governing the non-similar flow were solved by using an implicit finite difference scheme [7] as well as by local nonsimilarity method [8]. Merkin and Mahmood [9] obtained similarity solutions for the mixed convection flow over a vertical plate for the case of constant heat flux condition at the wall. The influence of the inclination angle and the Prandtl number on the mixed convection flow over an inclined flat plate for both constant wall temperature and heat flux conditions was examined by Wickern $[10,11]$. The effect of the surface mass transfer (suction/injection) on the mixed convection flow over a vertical porous plate for the constant wall temperature case was discussed by Watanabe [12]. The mixed convection flow at a two-dimensional stagnation point on a heated horizontal boundary was investigated by Amin and Riley [13]. The forced flow is a stagnation-point flow and the free convection part is due to a pressure gradient that is induced by the temperature variations along the boundary. The above studies deal with steady flows. However, in many problems of practical 
interest, the flow may be unsteady due to the change in the free stream velocity or in the surface temperature (heat flux) or in both.

When there is a sudden change in the velocity field, the inviscid flow is developed instantaneously, but the viscous flow near the wall is developed slowly and it becomes fully developed steady flow after certain instant of time. For small time the flow is dominated by the viscous forces and the unsteady acceleration, but for large time it is dominated by viscous forces, convective acceleration and pressure gradient. For small time the flow is generally independent of the conditions far upstream and at the leading edge or at the stagnation point, but for large time the flow depends on these conditions. The mathematical problem for small time is governed by the Rayleigh type of equation and for large time by the Blasius or Falkner-Skan type of equation.

The development of the boundary layer flow of a viscous fluid on a semi-infinite flat plate due to the impulsive motion of the free stream has been studied by Stewartson $[14,15]$, Hall [16], Dennis [17], Watkins [18] and Ingham [19]. The corresponding problem on a wedge was investigated by Smith [20], Nanbu [21], and Williams and Rhyne [22].

The flow and heat transfer development of the boundary layer on an impulsively rotating and translating sphere was considered by Ece [23], and Ozturk and Ece [24], respectively. In both cases the series solutions valid for small time were obtained. Kumari [25] studied the temporal development of flow and heat transfer over a wedge with a magnetic field caused by the impulsive motion of the free stream velocity and sudden change in the wall temperature. The governing boundary layer equations were solved numerically by using an implicit finite-difference scheme and the solutions are valid for all times.

Brown and Riley [26] considered the temporal development of the free convection flow past a suddenly heated semi-infinite vertical plate where the unsteadiness in the flow field is due to the step-change in the wall temperature. They showed that in the initial stage the flow is governed by one-dimensional flow model and a local solution describes the early stage of the departure from this. Also, an asymptotic solution describes the manner in which the final steady state is reached. Ingham [27] reconsidered the problem of Brown and Riley [26] by impulsively raising the wall temperature from its ambient temperature $T_{\infty}$ to $T_{w}\left(T_{w}=T_{\infty}+A x^{m}\right.$, where $A$ and $m$ are constants and $x$ is the distance measured from the leading edge of the plate) and obtained both numerical and asymptotic solutions. The numerical solution was found to match the large-and small-time asymptotic solutions only when the temperature increases along the plate. Recently, Seshadri et al. [28] have studied the unsteady mixed convection in the stagnation flow adjacent to a heated vertical surface where the unsteadiness is caused by the impulsive motion of the free stream velocity as well as sudden increase in the wall temperature. The parabolic partial differential equations governing the boundary layer flow have been solved by using an implicit finite-difference scheme starting from the initial steady state to the final steady state.

This paper investigates the unsteady mixed convection flow over a heated vertical flat plate. The unsteadiness is induced by impulsively creating motion in the ambient fluid and at the same time suddenly raising the wall temperature above the surrounding temperature or imposing a heat flux on the surface. The problem is formulated in such a way that at time $t=0$, it is governed by Rayleigh type of equation and as $t \rightarrow \infty$, it is governed by Blasius type of equation. Both constant wall temperature and constant wall heat flux conditions have been included in the analysis. The nonlinear coupled singular parabolic partial differential equations governing the boundary layer mixed convection flow have been solved numerically by using the implicit finite-difference scheme similar to that of Carter [29], and Williams and Rhyne [22]. The solutions have been obtained which are valid for all times starting from the initial steady state $(t=0)$ to the final steady state $\left(t^{*} \rightarrow \infty\right)$. The results have been compared with those of Wickern [10], Watanabe [12] and Williams and Rhyne [22].

\section{2}

\section{Problem formulation}

We consider a semi-infinite vertical plate which is placed in an ambient viscous fluid with uniform temperature $T_{\infty}$. The temperature of the plate is the same as that of the ambient fluid. Hence prior to the time $t<0$, there is no motion. At time $t=0$, the ambient fluid is impulsively moved with a constant velocity $u_{\infty}$ and at the same time the surface temperature is suddenly raised from $T_{\infty}$ to $T_{w}\left(T_{w}>T_{\infty}\right)$ or a constant heat flux condition $q_{w}$ is suddenly imposed on the surface of the plate. Subsequently, the same motion and surface temperature or heat flux are maintained. Fig. 1 shows the flow field over a vertical surface, where $x$ is the distance along the surface of the plate measured from the leading edge $x=0$ and $y$ is the distance normal to the surface. The buoyancy force arises due to the temperature difference between the surface and the fluid. The unsteadiness in the flow and temperature

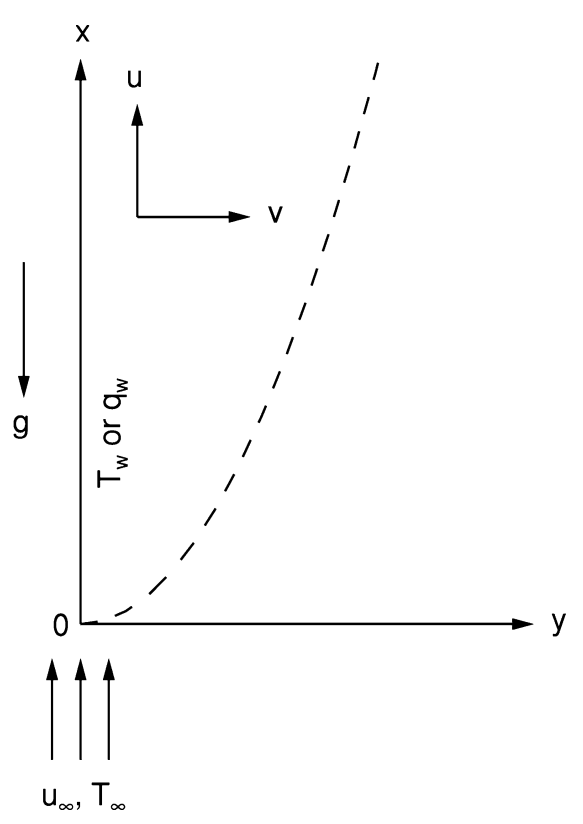

Fig. 1. Coordinate system 
fields is due to the impulsive motion of the ambient fluid and the sudden increase in the surface temperature or the sudden imposition of heat flux on the plate. The viscous dissipation term in the energy equation has been neglected. Applying the Boussinesq approximation, the boundary layer equations based on the conservation of mass, momentum and energy, governing the unsteady laminar incompressible mixed convection flow of the viscous fluid over a vertical plate can be expressed as

$u_{x}+v_{y}=0$,

$u_{t}+u u_{x}+v u_{y}=v u_{y y}+g \beta\left(T-T_{\infty}\right)$,

$T_{t}+u T_{x}+v T_{y}=\alpha T_{y y}$.

The initial conditions are given by

$u(x, y)=v(x, y)=0, \quad T(x, y)=T_{\infty}$ for $t<0$.

The boundary conditions for $t \geq 0$ are

$u(x, 0)=v(x, 0)=0, \quad u(x, \infty)=u_{\infty}, \quad T(x, \infty)=T_{\infty}$,

$T(x, 0)=T_{w}$ for the constant wall temperature case

(CWT case), $\partial T(x, 0) / \partial y=-q_{w} / K$ for the constant heat

flux case (CHF case).

Here $x$ and $y$ are, respectively, the distances along and perpendicular to the surface; $\mathrm{u}$ and $\mathrm{v}$ are the velocity components along $x$ and $y$ directions, respectively; $t$ is the time; $v$ is the kinematic viscosity; $g$ is the acceleration due to the gravity; $\beta$ is the volumetric coefficient of thermal expansion; $\alpha$ is the thermal diffusivity; the subscripts $t, x$ and $y$ denote derivatives with respect to $t, x$ and $y$, respectively; and the subscripts $w$ and $\infty$ denote conditions at the wall and in the free stream, respectively.

The problem due to the impulsive motion considered here should be formulated mathematically in such a way that for small times it should be governed by Rayleigh type of equations and for large times by Blasius type of equations. This implies that we have to select a scaling of $y$ coordinate which behaves like $y /(v t)^{1 / 2}$ for small times and as $y\left(u_{\infty} / v x\right)^{1 / 2}$ for large times. Further it is rather convenient to choose the time scale $\xi$ so that the region of time integration becomes finite. Williams and Rhyne [22] have found such transformations and they are expressed as

$\eta=\left(u_{\infty} / v x\right)^{1 / 2} y \xi^{-1 / 2}, \xi=1-\exp \left(-u_{\infty} t / x\right), t^{*}=u_{\infty} t / x$,

$u=\partial \psi / \partial y, v=-\partial \psi / \partial x, \psi(x, y, t)=\left(v x u_{\infty}\right)^{1 / 2} \xi^{1 / 2} f(\xi, \eta)$,

$T(x, y, t)-T_{\infty}=\left(T_{w}-T_{\infty}\right) \theta(\xi, \eta)$, (CWT case),

$T(x, y, t)-T_{\infty}=\left(q_{w} / K\right)\left(v x / u_{\infty}\right)^{1 / 2} \xi^{1 / 2} \theta(\xi, \eta)$ (CHF case).

Using (6) in equations (1)-(3), we find that equation (1) is identically satisfied and equations (2) and (3) for the CWT case reduce to

$$
\begin{aligned}
& f^{\prime \prime \prime}+2^{-1} \eta(1-\xi) f^{\prime \prime}+\left[2^{-1} \xi+(1-\xi) \log (1-\xi)\right] f f^{\prime \prime}+\lambda \xi \theta \\
& =\xi(1-\xi) \partial f^{\prime} / \partial \xi+\xi(1-\xi) \log (1-\xi)\left(f^{\prime} \partial f^{\prime} / \partial \xi-f^{\prime \prime} \partial f / \partial \xi\right),
\end{aligned}
$$

$$
\begin{aligned}
& \operatorname{Pr}^{-1} \theta^{\prime \prime}+2^{-1} \eta(1-\xi) \theta^{\prime}+\left[2^{-1} \xi+(1-\xi) \log (1-\xi)\right] f \theta^{\prime} \\
& =\xi(1-\xi) \partial \theta / \partial \xi+\xi(1-\xi) \log (1-\xi)\left(f^{\prime} \partial \theta / \partial \xi-\theta^{\prime} \partial f / \partial \xi\right),
\end{aligned}
$$

with boundary conditions

$f(\xi, 0)=f^{\prime}(\xi, 0)=0, \quad \theta(\xi, 0)=1, \quad f^{\prime}(\xi, \infty)=1$, $\theta(\xi, \infty)=0$,

where

$$
\begin{gathered}
\lambda=\mathrm{Gr}_{x} / \mathrm{Re}_{x}^{2}, \quad \mathrm{Gr}_{x}=g \beta\left(T_{w}-T_{\infty}\right) x^{3} / v^{2}, \\
\operatorname{Re}_{x}=u_{\infty} x / v, \quad \operatorname{Pr}=v / \alpha .
\end{gathered}
$$

Equations (7) and (8) for $\xi=0\left(t^{*}=0\right)$ and $\xi=1$ $\left(t^{\star} \rightarrow \infty\right)$ reduce to ordinary differential equations. For $\xi=0$, they are given by

$f^{\prime \prime \prime}+2^{-1} \eta f^{\prime \prime}=0$,

$\operatorname{Pr}^{-1} \theta^{\prime \prime}+2^{-1} \eta \theta^{\prime}=0$.

Similarly, for $\xi=1$ equations (7) and (8) are expressed as

$f^{\prime \prime \prime}+2^{-1} f f^{\prime \prime}+\lambda \theta=0$,

$\operatorname{Pr}^{-1} \theta^{\prime \prime}+2^{-1} f \theta^{\prime}=0$.

The boundary conditions for equations (11)-(14) are

$f(0)=f^{\prime}(0)=0, \quad \theta(0)=1, \quad f^{\prime}(\infty)=1, \quad \theta(\infty)=0$.

In a similar manner, for the constant heat flux case (CHF case), the equations corresponding to (7)-(15) can be expressed as

$$
\begin{aligned}
& f^{\prime \prime \prime}+2^{-1} \eta(1-\xi) f^{\prime \prime}+\left[2^{-1} \xi+(1-\xi) \log (1-\xi)\right] f f^{\prime \prime}+\lambda^{*} \xi^{3 / 2} \theta \\
& =\xi(1-\xi) \partial f^{\prime} / \partial \xi+\xi(1-\xi) \log (1-\xi)\left(f^{\prime} \partial f^{\prime} / \partial \xi-f^{\prime \prime} \partial f / \partial \xi\right),
\end{aligned}
$$

$$
\begin{aligned}
& \operatorname{Pr}^{-1} \theta^{\prime \prime}+2^{-1} \eta(1-\xi) \theta^{\prime}+2^{-1}[\xi+(1-\xi) \log (1-\xi)]\left(f \theta^{\prime}-f^{\prime} \theta\right) \\
& \quad-2^{-1}(1-\xi) \theta=\xi(1-\xi) \partial \theta / \partial \xi+\xi(1-\xi) \log (1-\xi) \\
& \quad\left(f^{\prime} \partial \theta / \partial \xi-\theta^{\prime} \partial f / \partial \xi\right)
\end{aligned}
$$

$f(\xi, 0)=f^{\prime}(\xi, 0)=0, \quad \theta^{\prime}(\xi, 0)=-1, \quad f^{\prime}(\xi, \infty)=1$,

$$
\theta(\xi, \infty)=0 \text {, }
$$

$f^{\prime \prime \prime}+2^{-1} \eta f^{\prime \prime}=0$,

$\operatorname{Pr}^{-1} \theta^{\prime \prime}+2^{-1} \eta \theta^{\prime}-2^{-1} \theta=0$,

$f^{\prime \prime \prime}+2^{-1} f f^{\prime \prime}+\lambda^{*} \theta=0$,

$\operatorname{Pr}^{-1} \theta^{\prime \prime}+2^{-1}\left(f \theta^{\prime}-f^{\prime} \theta\right)=0$,

$f(0)=f^{\prime}(0)=0, \quad \theta^{\prime}(0)=-1, \quad f^{\prime}(\infty)=1, \quad \theta(\infty)=0$,

where

$$
\lambda^{*}=G r_{x}^{*} / \mathrm{Re}_{x}^{5 / 2}, \quad \mathrm{Gr}_{x}^{*}=g \beta q_{w} x^{4} /\left(K v^{2}\right) .
$$


Here $\xi$ and $\eta$ are the transformed coordinates; $t^{*}$ is the dimensionless time; $\psi$ and $f$ are the dimensional and dimensionless stream functions, respectively; $\theta$ is the dimensionless temperature; $\mathrm{Pr}$ is the Prandtl number; $\mathrm{Gr}_{x}$ and $\mathrm{Gr}_{x}^{*}$ are the local Grashof numbers for the CWT and CHF cases, respectively; $\lambda$ and $\lambda^{*}$ are the buoyancy parameters for the CWT and CHF cases, respectively; $\mathrm{Re}_{x}$ is the local Reynolds number; $q_{w}$ is the surface heat flux; $K$ is the thermal conductivity; and prime denotes derivative with respect to $\eta$.

It may be noted that equations (11) and (12) under conditions (15) and equations (19) and (20) under conditions (23) represent the early flow development equations for the CWT and CHF cases, respectively and these equations are of Rayleigh type. Similarly, equations (13) and (14) under condition (16), and equations (21) and (22) under conditions (23) represent the final steady state for the CWT and CHF cases, respectively, and they are of Blasius type. Further, equations (13) and (14) under conditions (15) are identical to those of Watanabe [12]. Also, equations (13)-(15) and equations (21)-(23) are identical to those of Wickern [10]. In the absence of the buoyancy force $\left(\lambda=\lambda^{*}=0\right)$, equation (7) under conditions (9) or equation (16) under condition (17) is identical to that of Williams and Rhyne [22] when we put the pressure gradient parameter $m=0$ in their equation.

\section{3}

\section{Analytical solution}

The solutions of the linear equations (11) and (12) under conditions (15) can be expressed as

$f=\eta \operatorname{erf}(\eta / 2)-(\pi)^{-1 / 2}\left[1-\exp \left(-\eta^{2} / 4\right)\right]$

$\theta=\operatorname{erfc}\left(\operatorname{Pr}^{1 / 2} \eta / 2\right)$.

Hence

$f^{\prime \prime}(0)=(\pi)^{-1 / 2}, \quad \theta^{\prime}(0)=-(\operatorname{Pr} / \pi)^{1 / 2}$.

It may be noted that the solution of (19) under conditions (23) is also given by (25a). Also, the solution of equation (20) under conditions (23) can be expressed in the form [30]

$$
\begin{aligned}
\theta= & -\exp \left[-\operatorname{Pr}\left(1+2^{-1} \operatorname{Pr}\right) \eta^{2} / 8\right] \\
& {\left[\frac{2}{\operatorname{Pr}} \frac{A}{B}{ }_{1} F_{1}\left(\frac{1}{4}\left(1+\frac{3}{2} \operatorname{Pr}\right), \frac{1}{2}, \frac{\operatorname{Pr}^{2} \eta^{2}}{8}\right)\right.} \\
& \left.+\eta_{1} F_{1}\left(\frac{3}{4}\left(1+\frac{\operatorname{Pr}}{2}\right), \frac{3}{2}, \frac{\operatorname{Pr}^{2} \eta^{2}}{8}\right)\right],
\end{aligned}
$$

where

$A=\Gamma(1 / 2) / \Gamma(3(2+\operatorname{Pr}) / 8), B=2^{-1 / 2} \Gamma(-1 / 2) / \Gamma(2+3 \operatorname{Pr}) / 8$,

${ }_{1} F_{1}(a, c, x)=\sum_{j=0}^{\infty} C_{j} x^{j}, C_{j}=\frac{a(a+1) \cdots(a+j-1) 1}{c(c+1) \cdots(c+j-1) j !}$,

${ }_{1} F_{1}(1+a-c, 2-c, x)=\sum_{j=0}^{\infty} D_{j} x^{j}$,
$D_{j}=\frac{(1+a-c)(2+a-c) \cdots(1+a-c+j-1)}{(2-c)(3-c) \cdots\left(2-c+j-^{\prime} 1\right) x} \frac{1}{j !}$,

$a=(2+\operatorname{Pr}) / 8, \quad c=1 / 2, \quad x=\operatorname{Pr}^{2} \eta^{2} / 8$.

Here ${ }_{1} F_{1}(a, c, x)$ is the confluent hypergeometric series and $\Gamma$ denotes the gamma function. It may be remarked that the numerical results are found to agree with the analytical results at least up to three decimal places.

\section{4}

\section{Numerical method}

Equations (7) and (8) are the coupled nonlinear partial differential equations and they under conditions (9)-(15) can be solved by using an implicit finite difference scheme. Equations (7) and (8) can be rewritten as

$H_{1}^{\prime \prime}+a_{1} H_{1}^{\prime}+a_{2} H_{2}=a_{3} \partial H_{1} / \partial \xi$

$\operatorname{Pr}^{-1} H_{2}^{\prime \prime}+a_{1} H_{2}^{\prime}=a_{3} \partial H_{2} / \partial \xi$

where

$$
\begin{aligned}
a_{1}= & 2^{-1} \eta(1-\xi)+2^{-1} \xi f+(1-\xi) \log (1-\xi) f \\
& +\xi(1-\xi) \log (1-\xi) \partial f / \partial \xi, \\
& a_{2}=\lambda \xi, \quad a_{3}=\xi(1-\xi)\left[1+\log (1-\xi) H_{1}\right], H_{1}=f^{\prime}, H_{2}=\theta .
\end{aligned}
$$

The boundary conditions (9) can be expressed as

$$
\begin{aligned}
H_{1}(\xi, 0) & =f(\xi, 0)=0, \quad H_{2}(\xi, 0)=1, H_{1}(\xi, \infty) \\
& =1, \quad H_{2}(\xi, \infty)=0 .
\end{aligned}
$$

It may be noted that $a_{3}$ which is the coefficient of $\partial H_{i} /$ $\partial \xi, i=1,2$ in equations (28) and (29) is positive when $\xi<$ $1-e^{-1}$, since $H_{i}>0$ in $0<\eta<\eta_{\infty}$, but it becomes negative for some $\eta$ when $\xi>1-e^{-1}$. When $a_{3}>0$, equations (28) and (29) under conditions (31) are parabolic partial differential equations and are well-posed. When $a_{3}<0$, the problem is not well posed and forward integration method fails [22]. Such equations are known as singular parabolic partial differential equations [19]. Physically, the change in the sign of $a_{3}$ is attributed to the change in the direction of flow as explained in [19].

In order to overcome the above difficulties, we have used either forward or backward difference in $\xi$ direction consistent with the direction of the flow. In $\eta$-direction, we have used the central difference scheme. The present method of solution is based on the method used by Carter [29] and its detailed description is given by Williams and Rhyne [22]. Figure 2 shows the computational region and the behaviour of the coefficient $a_{3}$.

In a similar manner, equations (16) and (17) under conditions (18)-(23) have also been solved. We have carried out the sensitivity analysis on the effects of the step size $\Delta \eta$ and $\Delta \xi$, and the edge of the boundary layer $\eta_{\infty}$ on the solution. Finally we have chosen $\Delta \eta=0.05, \Delta \xi=0.01$, $\eta_{\infty}=10$. 


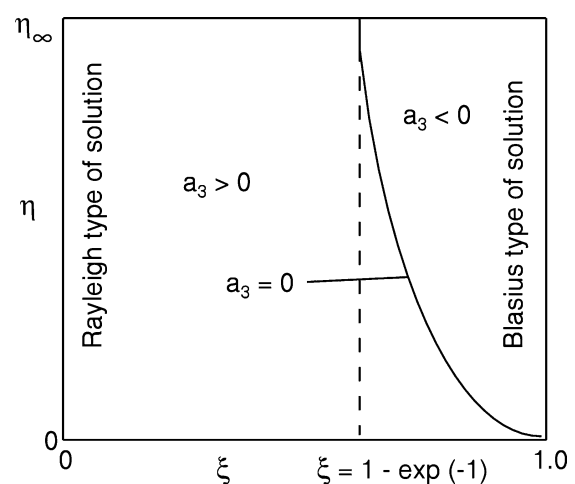

Fig. 2. Schematic representation of computational domain

\section{5}

\section{Results and discussion}

Equations (7) and (8) under conditions (9)-(15) and equations (16) and (17) under conditions (18)-(23) have been solved by the implicit finite-difference scheme described earlier. In order to assess the accuracy of our method, we have compared our velocity and temperature profiles for the final steady state $(f(\eta), \theta(\eta))$ corresponding to the CWT case for $\xi=1, \operatorname{Pr}=0.73$ with those of Watanabe [12]. We have also compared our skin friction and heat transfer results $\left(f^{\prime}(0),-\theta^{\prime}(0)\right.$ or $\left.(\theta(0))^{-1}\right)$ for both the CWT and CHF cases when $\xi=1, \operatorname{Pr}=0.72$ with those of Wickern [10]. In all the cases the results are found to be in very good agreement. For the sake of brevity, the comparison is not presented here. Further, we have compared our surface shear stress $f^{\prime}(\xi, 0)$ in the range $0 \leq \xi$ $\leq 1$ (i.e., from the early flow development to the final steady state) in the absence of the buoyancy force $(\lambda=0)$ with the corresponding results of Williams and Rhyne [22]. The results are in excellent agreement. The comparison is shown in Fig. 3 .

Figures 3 and 4 show the variation of the surface skin friction and heat transfer parameters $\left(f^{\prime}(\xi, 0),-\theta^{\prime}(\xi, 0)\right)$ with time $\xi$ for the constant wall temperature case (CWT case) and for $\operatorname{Pr}=0.7$. For a given $\xi>0$, the skin friction and heat transfer parameters $\left(f^{\prime}(\xi, 0),-\theta^{\prime}(\xi, 0)\right)$ increase with the buoyancy parameter $\lambda(\lambda>0)$. The reason for this

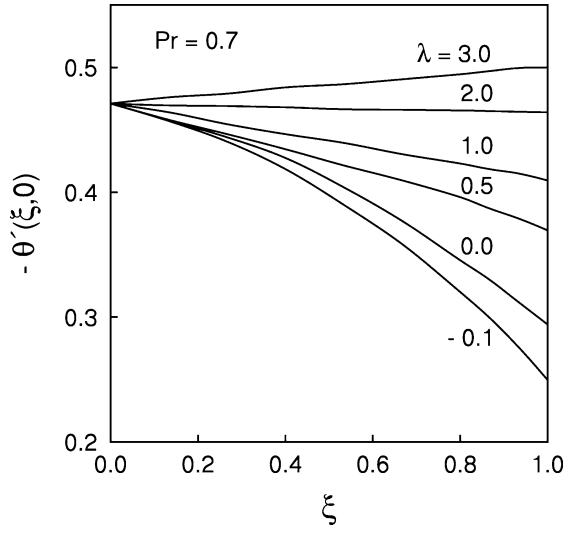

Fig. 4. Variation of the surface heat transfer for the CWT case $\left(-\theta^{\prime}(\xi, 0)\right)$ with time $\xi$ when $\operatorname{Pr}=0.7$

behaviour is that the positive buoyancy force acts like a favourable pressure gradient which accelerates the flow field. This results in thinner momentum and thermal boundary layers. Consequently, the velocity and temperature gradients at the wall increase with $\lambda$. Since the motion is created impulsively at $\xi=0$, the flow and thermal fields are governed by Rayleigh type of equations and for $\lambda<\lambda_{0}$, the surface shear stress and heat transfer are maximum at $\xi=0$ and they decrease with increasing $\xi$. For the surface shear stress $f^{\prime}(\xi, 0), \lambda_{0}=0.24$ and for the surface heat transfer $-\theta^{\prime}(\xi, 0), \lambda_{0}=2.28$. On the other hand, for $\lambda>\lambda_{0}$ both $f^{\prime}(\xi, 0)$ and $-\theta^{\prime}(\xi, 0)$ increase with $\xi$. The reason for this trend can be explained as follows. Since the buoyancy parameter $\lambda$ is multiplied by $\xi$, the effect of $\lambda$ vanishes at $\xi=0$ and increases with $\xi$. As mentioned earlier the positive buoyancy force accelerates both velocity and thermal fields, $f^{\prime}(\xi, 0)$ and $-\theta^{\prime}(\xi, 0)$ increase with $\xi$ when $\lambda>\lambda_{0}$. Further, the effect of the buoyancy force is more pronounced on the surface shear stress $f^{\prime}(\xi$, $0)$ than on the surface heat transfer $-\theta^{\prime}(\xi, 0)$, because the buoyancy parameter $\lambda$ occurs explicitly in the momentum equation but its effect on the energy equation is indirect (see Eqs. (7) and (8)).

Figures 5 and 6 present the variation of the surface shear stress $\left(f^{\prime}(\xi, 0)\right)$ and the surface heat transfer

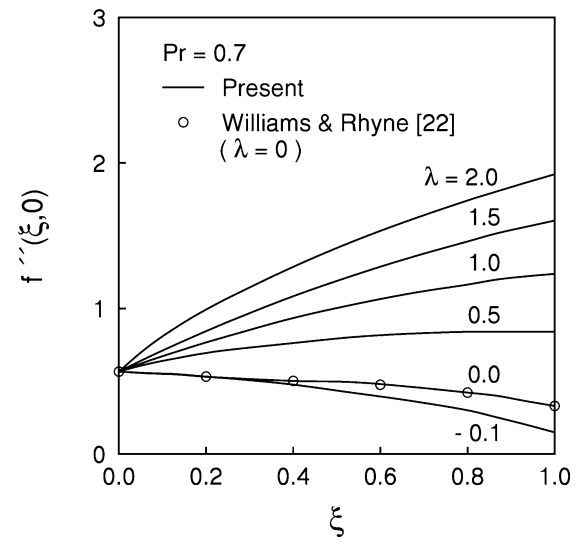

Fig. 3. Variation of the surface shear stress for the CWT case $\left(f^{\prime}(\xi, 0)\right)$ with time $\xi$ when $\operatorname{Pr}=0.7$

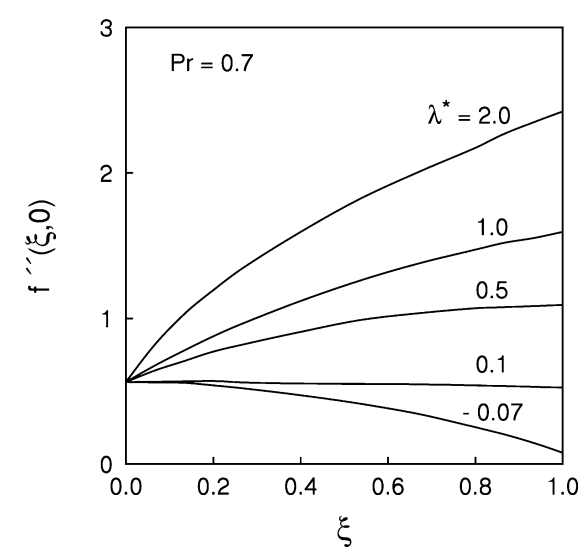

Fig. 5. Variation of the surface shear stress for the CHF case $\left(f^{\prime}(\xi, 0)\right)$ with time $\xi$ when $\operatorname{Pr}=0.7$ 


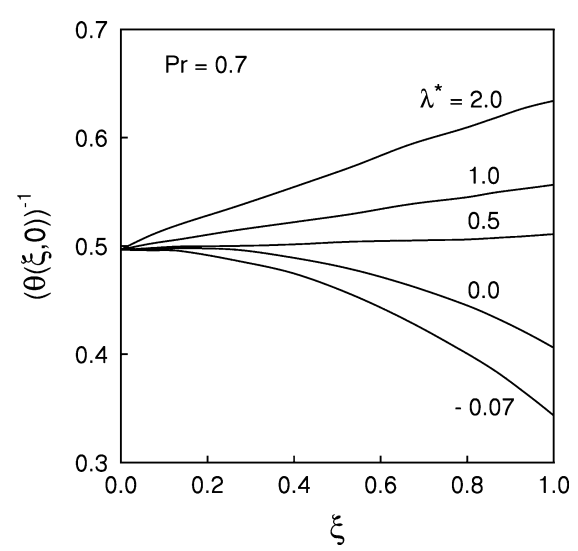

Fig. 6. Variation of the surface heat transfer for the CHF case $\left((\theta(\xi, 0))^{-1}\right)$ with time $\xi$ when $\operatorname{Pr}=0.7$

$(1 / \theta(\xi, 0))$ with time $\xi$ for the constant wall heat flux case (CHF case) when $\mathrm{Pr}=0.7$. Since the behaviour is qualitatively similar to that of the CWT case, it is not discussed here. However, the surface shear stress and heat transfer for the CHF case, in general, are higher than those of the CWT case. The reason for this trend is that for the CHF case the wall temperature is higher than that of the CWT case. Hence the value of the buoyancy force term $\left(\lambda^{*} \xi^{3 / 2} \theta\right)$ is more than that of the CWT case for the same values of $\lambda$, $\lambda^{*}$ and $\xi$. The higher buoyancy force implies thinner momentum and thermal boundary layers. Consequently, the surface shear stress and heat transfer for the CHF case are higher than those of the CWT case.

\section{6}

\section{Conclusions}

There is a smooth transition from the early flow development to the final steady state. The surface shear stress and the heat transfer increase or decrease with time when the buoyancy parameter is greater or less than a certain value. Both the surface shear stress and the heat transfer increase with the buoyancy parameter after the start of the motion. The surface shear stress and the heat transfer for the constant heat flux case are greater than those of the constant wall temperature case.

\section{References}

1. Sparrow EM; Eichhom R; Gregg JC (1959) Combined forced and free convection in a boundary layer flow. Phys Fluids 2: 319-328

2. Sparrow EM; Gregg JC (1959) Buoyancy effects in forced convection flow and heat transfer. J Appl Mech 26: 133-138

3. Szewczyk AA (1964) Combined forced and free convection laminar flow. J Heat Transfer 86: 501-507
4. Merkin JH (1969) The effect of buoyancy forces on the boundary layer flow over a semi-infinite vertical plate in a uniform stream. J Fluid Mech 35: 439-450

5. Oosthuizen PH; Hart H (1973) A numerical study of laminar combined convective flow over a flat plate. J Heat Transfer 95: 6063

6. Mucoglu A; Chen TS (1979) Mixed convection on inclined surfaces. J Heat Transfer 101: 422-426

7. Cebeci T; Bradshaw P (1977) Momentum Transfer in Boundary Layers. Hemisphere Publishing Corp, Washington DC

8. Sparrow EM; Yu HS (1971) Local nonsimilarity boundary layer solutions. J Heat Transfer 93: 328-334

9. Merkin JH; Mahmood T (1989) Mixed convection boundary layer similarity solution: prescribed heat flux. ZAMP 40: 61-68

10. Wickern G (1991) Mixed convection from an arbitrarily inclined semi-infinite flat plate-I. The influence of inclination angle. Int J Heat Mass Transfer 34: 1935-1945

11. Wickern G (1991) Mixed convection from an arbitrarily inclined semi-infinite flat plate-II. The influence of the Prandtl number. Int J Heat Mass Transfer 34: 1947-1957

12. Watanabe T (1991) Forced free mixed convection boundary layer flow with uniform suction or injection. Acta Mechanica 89: 123132

13. Amin N; Riley N (1995) Mixed convection at a stagnation point. Quart J Mech Appl Math 48: 111-121

14. Stewartson K (1951) On the impulsive motion of a flat plate in a viscous fluid, Part I. Quart J Mech Appl Math 4: 162-198

15. Stewartson K (1973) On the impulsive motion of a flat plate in a viscous fluid, Part II. Quart J Mech Appl Math 22: 143-152

16. Hall MG (1969) The boundary layer over an impulsively started flat plate. Proc Roy Soc 310A: 401-414

17. Dennis SCR (1972) The motion of a viscous fluid past an impulsively started semi-infinite flat plate. J Inst Math Appl 10: 105-117

18. Watkins CB (1975) Heat transfer in the boundary layer over an impulsively started flat plate. J Heat Transfer 97: 482-484

19. Ingham DB (1977) Singular parabolic partial differential equations that arise in impulsive motion problems. J Appl Mech 44: 396-400

20. Smith SH (1967) The impulsive motion of a wedge in a viscous fluid. ZAMP 18: 508-522

21. Nanbu K (1976) Unsteady Falker-Skan flow. ZAMP 22: 1167-1172

22. Williams JC; Rhyne TH (1980) Boundary layer development of a wedge impulsively set into motion. SIAM J Appl Math 38: 215-224

23. Ece MC (1992) An initial boundary layer flow past a translating and spinning rotational symmetric body. J Eng Math 26: 415-423

24. Ozturk A; Ece MC (1995) The unsteady forced convection flow transfer from a translating and spinning body. J Energy Resour Technol 117: 318-323

25. Kumari M (1997) Development of flow and heat transfer on a wedge with a magnetic field. Arch Mech 49: 977-990

26. Brown SN; Riley N (1973) Flow past a suddenly heated vertical plate. J Fluid Mech 59: 225-237

27. Ingham DB (1985) Flow past a suddenly heated vertical plate. Proc Roy Soc 402A: 109-134

28. Seshadri R; Sreeshylan N; Nath G (2002) Unsteady mixed convection flow in the stagnation region of a heated vertical plate due to impulsive motion. Int J Heat Mass Transfer 45: 1345-1352

29. Carter JE (1975) Inverse solutions for laminar boundary layer flows with separation and attachment. NASA TR-R447, Washington DC

30. Whittacker ET; Watson GN (1963) Modern Analysis Cambridge University Press, London 